\title{
LUCES Y SOMBRAS EN TORNO A LA CONCILIACIÓN DE LAS FAMILIAS CON HIJOS E HIJAS CON NEAE: UN ESTUDIO EN GALICIA
}

\author{
LIGHTS AND SHADOWS OF RECONCILIATION IN FAMILIES WITH CHILDREN \\ WITH SPECIFIC NEEDS OF LEARNING SUPPORT: STUDY IN GALICIA
}

\section{LUZES E SOMBRAS EM TORNO DA CONCILIAÇÃO DAS FAMILIAS COM FILHOS E FILHAS COM NEAE: UM ESTUDO NA GALIZA}

\author{
Laura VARELA CRESPO*, M. Esther MARTÍNEZ FIGUEIRA** \& \\ Ángela L. de VALENZUELA BANDÍN* \\ Universidad de Santiago de Compostela*, Universidad de Vigo**
}

PALABRAS CLAVE: conciliación vida laboral

familiar y personal enseñanza primaria necesidades

específicas de apoyo educativo

servicios

comunitarios
Fecha de recepción: 02.111.2018 Fecha de revisión: 05.111.2018 Fecha de aceptación: 23.V.2018

CONTACTO CON LOS AUTORES: LAURA VARELA CRESPO: Universidad de Santiago de Compostela. Departamento de Pedagogía y Didáctica. Facultad de Ciencias de la Educación-Campus vida. Rúa Prof. Vicente Fráiz Andón, s/n. 15782, Santiago de Compostela. E-mail: laura.varela@usc.es 


\begin{tabular}{|c|c|}
\hline $\begin{array}{l}\text { KEY WORDS: } \\
\text { reconciliation of } \\
\text { working, family } \\
\text { and personal life } \\
\text { primary education } \\
\text { specific needs of } \\
\text { learning support } \\
\text { community services }\end{array}$ & $\begin{array}{l}\text { ABSTRACT: The diversity of factors and implications that are inherent to reconciliation of } \\
\text { working, family and personal times involves the perception of limitations or difficulties in the } \\
\text { arduous task of reconciling on the one hand and the identification of possible ways and so- } \\
\text { cial-pedagogical supports to facilitate it on the other. The goal of the present article is to iden- } \\
\text { tify the obstacles and alternatives for families with children with specific needs of learning } \\
\text { support (SNLS) attending primary school in Galicia regarding the issue of harmonizing daily } \\
\text { times. This exploratory and descriptive study belongs to a wider investigation -entitled Concil- } \\
\text { ia_d@s- based on the implementation of an ad hoc questionnaire, in a target group consisting } \\
\text { of families of the student body of Primary Education ( } n=2037 \text {; e }=2,2 \% \text {; confidence= } 95,5 \% \text { ) in } \\
\text { the Autonomous Community of Galicia. The outcome showed the difficulties for the organi- } \\
\text { zation of times encountered by families with children with SNLS ( } n=127 \text { ) due to their need of } \\
\text { special attention, as well as the resources and social-pedagogical alternatives that could guar- } \\
\text { antee a better distribution of times, promoting childhood education and the construction of a } \\
\text { more inclusive society. Analyzing the data obtained, we noted "lights" and "shadows" affecting } \\
\text { families in Galicia -specifically those families with children with SNLS- for managing their } \\
\text { times and supporting the process of social and educational inclusion of childhood. }\end{array}$ \\
\hline $\begin{array}{l}\text { PALAVRAS-CHAVE: } \\
\text { conciliação vida } \\
\text { profissional } \\
\text { familiar e pessoal } \\
\text { ensino primário } \\
\text { necessidades } \\
\text { específicas de } \\
\text { apoio educacional } \\
\text { serviços } \\
\text { comunitários }\end{array}$ & $\begin{array}{l}\text { RESUMO: A diversidade de fatores e implicaçães inerentes à conciliação dos tempos de tra- } \\
\text { balho, familiares e pessoais, implica a perceção de limitações ou dificuldades na árdua tarefa } \\
\text { de conciliação, bem como o reconhecimento de apoios pedagógico-sociais que a facilitem. O } \\
\text { objetivo do trabalho que é apresentado é conhecer os obstáculos e as alternativas que as fa- } \\
\text { mílias galegas com filhos e filhas com necessidades específicas de apoio educacional (NEAE) } \\
\text { em escolas de ensino primário identificam em torno da harmonização de seus tempos diários. } \\
\text { Este estudo exploratório e descritivo faz parte de uma pesquisa mais ampla -denominada } \\
\text { Concilia_d @ s-, com base na aplicação de um questionário, criado e validado ad hoc, às famí- } \\
\text { lias dos alunos do ensino primário (n=2O37; e=2,2\%; confiança=95,5\%) da comunidade autóno- } \\
\text { ma da Galiza. Os resultados mostram as dificuldades que as famílias com crianças com NEAE } \\
\text { (n=127) devem enfrentar no que se refere à organização dos seus cuidados, relativamente aos } \\
\text { recursos e às alternativas pedagógicas-sociais que garantam uma melhor conciliação dos seus } \\
\text { tempos, favorecendo a atenção educacional da infância e a construção de uma sociedade } \\
\text { mais inclusiva. Com base na reflexão teórica feita e na análise dos dados obtidos, foi possível } \\
\text { cohecer algumas das "luzes" e "sombras" que influenciam as famílias galegas -particularmente } \\
\text { naquelas com crianças com NEAE- na conciliação dos seus tempos e no apoio ao processo } \\
\text { de inclusão social e educacional das crianças. }\end{array}$ \\
\hline
\end{tabular}

\section{Introducción}

La conciliación de los tiempos personales, familiares y laborales asociada a un reparto corresponsable de las tareas dentro y fuera del hogar (Caballo, Gradaílle \& Merelas, 2012; Gómez \& Jiménez, 2015) constituye a día de hoy uno de los principales desafíos que las sociedades han de afrontar para garantizar el bienestar de las familias, especialmente de aquellas cuyos hijos e hijas presentan Necesidades Específicas de Apoyo Educativo -en adelante NEAE- (artículo 71, LOMCE, 2013), ofreciendo a la infancia una atención educativa de calidad y evitando la subordinación de los tiempos libres de los niños y niñas a la mera custodia. Las NEAE, según la Ley Orgánica 8/2013 para la mejora de la calidad educativa (LOMCE), se clasifican en: necesidades educativas especiales (NEE); dificultades específicas de aprendizaje (DEA); trastorno por déficit de atención e hiperactividad (TDAH); altas capacidades intelectuales (AC); incorporación tardía (IT); o condiciones personales o de historia escolar (CP-HE).

Cabe afirmar que el colectivo de alumnado con NEAE es heterogéneo y las realidades de sus familias diversas; de ahí que las posibilidades de conciliación dependan -además de factores como la edad, los servicios comunitarios disponibles, etc.- del tipo de necesidad educativa (Martínez-Figueira, Varela \& De Valenzuela, 2016). Las estrategias y medidas de conciliación a las que las familias tienen acceso repercuten en su calidad de vida, puesto que su bienestar o malestar estará condicionado por la presencia efectiva o la carencia de servicios y recursos orientados a favorecer la organización de sus tiempos cotidianos. Disponer de medidas tales como la reducción de jornada, las excedencias, las licencias o permisos de paternidad o maternidad, la flexibilización, adaptación o reasignación de servicios y horarios en función de las necesidades, etc., promovidas desde el ámbito sociolaboral constituye un logro importante respecto al desarrollo personal de todos los integrantes de la unidad familiar y a su plena participación en la sociedad.

Según Mingo y Escudero (2008, p. 296):

los horarios de trabajo propios de las empresas, la falta de flexibilidad laboral, la forma de distribución de los permisos de vacaciones, la descoordinación entre los "tiempos" de los trabajos y los de las escuelas, la competitividad, los cambios en la composición 
y estructura de las familias -muchas de ellas monoparentales-, el aislamiento y pérdida de redes sociales y de apoyo, dificultan la confluencia de un conjunto de intereses contrapuestos. De un lado, los adultos deben afrontar tensiones importantes en las relaciones trabajo-familia $y$, de otro, las formas adoptadas para la organización de los tiempos resultan incompatibles con las necesidades de la infancia, repercutiendo negativamente -y en ocasiones vulnerandosu derecho a educarse en los diversos escenarios de su vida cotidiana, a disfrutar de experiencias de ocio y a compartir más tiempo con sus progenitores.

Entre las múltiples estrategias que utilizan las familias para conciliar sus tiempos, el papel de los abuelos/as resulta fundamental para compatibilizar el cuidado de los menores que presentan alguna necesidad educativa con los horarios laborales de los adultos (Díaz, Gil \& Moral, 2010); una situación generalizada en el contexto español donde la familia extensa continúa siendo un apoyo esencial en el mantenimiento del bienestar. Investigaciones como la de Jung y Appelbaum (2010) revelan que los padres y madres cuyos hijos/as presentan alguna discapacidad o necesidad educativa tienen más probabilidad de trabajar a tiempo parcial y de recibir menos beneficios; experimentando con frecuencia niveles de estrés, depresión o ansiedad que exigen tratamiento médico. Todo ello implica una vivencia familiar de sobrecarga y de renuncias personales (Vives, 2007; Liñán, 2013), ya que la unidad familiar necesita el sustento económico y los beneficios que aporta el desarrollo profesional, a la vez que debe responder a las tareas de cuidado y atención de la infancia.

Asimismo, no se ha de olvidar que en el caso de las familias con hijas e hijos con NEAE, la función educativa que ejercen los padres y madres exige un seguimiento y acompañamiento a la infancia de mayor intensidad que el que requieren otras niñas y niños de su misma edad, a fin de garantizar el máximo desarrollo de sus potencialidades y su plena inclusión social; una labor que difícilmente se puede llevar a cabo si los adultos no disponen del tiempo suficiente ni de los apoyos educativos y sociales apropiados.

Durante la década de los noventa, la investigación en torno a la percepción de la satisfacción con la vida cotidiana de las familias con hijos/as con NEAE se centraba fundamentalmente en el impacto de la discapacidad, en sí misma, en el bienestar y estrés familiar que generaba, sin llevarse a cabo un análisis de la situación desde una perspectiva integral (Weiss, Marvin \& Pianta, 1997; Browne \& Bramster, 1998; Rubio, Berg-Weger \& Tebb, 1999). Actualmente, los estudios tienen la finalidad de determinar los niveles de satisfacción e insatisfacción de los padres y madres con su vida, tratando de identificar y comprender las necesidades que perciben según variables sociodemográficas como: el tipo de discapacidad, el género, la edad, la tipología de familia o el estatus socioeconómico.

Desde esta perspectiva, surgen trabajos que visualizan a la familia en su conjunto como una unidad de apoyo en la búsqueda de logros que respondan a sus necesidades reales y a sus expectativas (Park, Turnbull \& Turnbull, 2002; Schalock \& Verdugo, 2002), adoptando una orientación focalizada en los factores de éxito y puntos fuertes (Trute, Hiebert-Murphy \& Levine, 2007; Córdoba-Andrade, Gómez-Benito y Verdugo-Alonso, 2008; Algood, Harris \& Sung, 2013). Complementariamente, se entiende que cualquier cambio que se lleve a cabo en materia de conciliación debe enfocarse desde una perspectiva compleja y holística, incluyendo el derecho al tiempo como un elemento clave para la satisfacción familiar (Caballo, Caride \& Meira, 2011; Sánchez, 2011).

En suma, los hallazgos de los últimos años evidencian la necesidad de ampliar la cobertura de las familias, la importancia de los recursos en el lugar de trabajo, así como de los servicios comunitarios para afrontar el desafío de atender a la diversidad. Además, en lo que respecta al soporte social, en un estudio comparativo entre familias de niñas y niños con y sin NEAE en el que ambos grupos cuentan con redes de apoyo similares (Felizardo, Ribeiro, Cardoso \& Campos, 2014), se constata que para los progenitores de hijas e hijos con NEAE estas redes resultan menos satisfactorias en el abordaje de necesidades de tipo emocional e instrumental. Asimismo, en la atención a la infancia con NEAE, numerosos estudios señalan que las madres presentan menos satisfacción y mayores niveles de estrés que los padres (Gerstein, Crnic, Blacher \& Baker, 2009; Lee, Park \& Recchia, 2015; Küçük \& Küçük, 2017), debido a que son las mujeres quienes asumen mayoritariamente las tareas de cuidado asociadas al ámbito doméstico, adaptándolas a sus tiempos personales y laborales (Caballo, Gradaílle \& Merelas, 2012; Maganto \& Etxeberria, 2012).

Algunas de las repercusiones que se derivan de esta situación es que ellas tienen respecto a los hombres mayores dificultades para compatibilizar los tiempos personales, familiares y laborales, así como para avanzar a nivel profesional. De ahí que se perpetúen situaciones de desigualdad al no alcanzarse la anhelada corresponsabilidad debido, entre otras causas, a la falta de conciencia social y a la ausencia de una regulación normativa eficiente (Merino, 2015). Sobre este último aspecto, en el sistema jurídico español, el derecho a la 
conciliación de la vida personal, familiar y laboral es considerado, "como un derecho de igualdad tendente a realizar la corresponsabilidad entre mujeres y hombres en la asunción de obligaciones familiares y erradicar las discriminaciones de la mujer en el mercado laboral y en las relaciones familiares" (Calvo \& Picontó, 2014, p. 98).

La primera medida legislativa aprobada en España en esta materia fue la Ley 39/1999, de 5 de noviembre, para promover la conciliación de la vida familiar y laboral de las personas trabajadoras, que se amplió, posteriormente, con la Ley Orgánica 3/2007, de 22 de marzo, para la igualdad efectiva de mujeres y hombres. En el contexto gallego, ámbito geográfico de la investigación que se presenta, destacan la Ley 7/2004, de 16 de julio, gallega para la igualdad de mujeres y hombres y la Ley 2/2007, de 28 de marzo, del trabajo en igualdad de las mujeres de Galicia, que incide en la necesidad de la inserción laboral de las mujeres como un pilar esencial.

La existencia de este marco normativo constituye un avance significativo respecto a la igualdad de oportunidades, al visibilizar una problemática latente desde hace tiempo. Sin embargo, dicho marco se centra casi de modo exclusivo en favorecer medidas en el ámbito laboral, obviándose otras circunstancias que condicionan -en función del género- el disfrute de una vida de calidad, principalmente, respecto a la distribución de los tiempos cotidianos (Merino, 2015). Por ello, "el trasfondo de este escenario legislativo ha sido objeto de críticas al responder a la lógica productiva y mostrarse ciego al género" (Moreno, 2009, p. 48).

El arraigo al modelo tradicional, basado en la lógica de la producción y la reproducción -asignadas a los hombres y a las mujeres respectivamente- repercute en que el ejercicio de los derechos de conciliación no se corresponda con la promoción de la corresponsabilidad y la igualdad a las que se alude en las medidas legislativas. Se pone así en cuestión la eficacia del discurso normativo sobre la igualdad, discordante con su concreción en hechos, pudiendo afirmarse que nos hallamos ante una problemática de cambio social, donde las transformaciones sociales y culturales no se suceden al compás del cambio legislativo, sino que van más lentas (Rivero, 2005; Calvo \& Picontó, 2014).

Un tema-problema, el de la conciliación de los tiempos de las familias con hijos e hijas con NEAE, que hace ineludible recuperar la visión social y educativa transformadora que ofrece la Pedagogía Social a partir de una lectura multidimensional del fenómeno que implique

una reflexión sobre el actual modelo productivo y los tiempos laborales, sobre la corresponsabilidad de los diferentes integrantes de las unidades familiares en la realización de las tareas domésticas y en las del cuidado; así como en la necesidad de contar con un tiempo de libre disposición, fundamental para el desarrollo integral de las personas y para la participación en la vida comunitaria (Caballo, Caride \& Meira, 2011, p. 19).

\section{Metodología}

El objetivo del presente trabajo es conocer las dificultades y alternativas que perciben las familias con hijos e hijas con Necesidades Específicas de Apoyo Educativo, que cursan Educación Primaria en la Comunidad Autónoma de Galicia, en relación con la conciliación de sus tiempos personales, familiares y laborales. Los resultados que se presentan forman parte de un proyecto de investigación más amplio denominado Concilia_d@s, orientado a conocer e interpretar los tiempos cotidianos de las familias del alumnado que cursa Educación Primaria en Galicia, en función de sus contextos de residencia habitual (rural y urbano), los tiempos escolares (calendarios y horarios lectivos) y las actividades de ocio que realizan. Todo ello, sin obviar la valoración de la incidencia que tienen distintas variables como el género, las condiciones laborales de los padres/madres o los procesos de socialización (inclusión/exclusión).

En concreto, en este trabajo, se hace referencia a las familias con hijos e hijas que presentan alguna Necesidad Específica de Apoyo Educativo $(n=127)$, representando un $6,2 \%$ de la distribución de la muestra total $(n=2037)$. Teniendo en cuenta la clasificación que del término NEAE hace la actual legislación española en materia educativa -LOMCE en su artículo 71.2-, se han distribuido las familias de la muestra que tienen hijos e hijas con alguna NEAE en función de los siguientes subtipos: $40,3 \%(n=50)$ con TDAH, $28,2 \%(n=35)$ con NEE, $12,1 \%(n=15)$ con DEA, $4 \%(n=4)$ con AC y $3,5 \%(n=4)$ con CP-HE; en cuanto a las familias del alumnado que se han incorporado tardíamente al sistema educativo (IT) no existe representatividad. Además, se ha incluido el grupo de "otros" $(12,1 \%, n=15)$, para hacer referencia a las familias que -aun reconociendo que sus hijos e hijas presentan NEAE- no especifican la tipología.

Considerando que la población de referencia son las familias con menores escolarizados en Educación Primaria durante el curso académico 2014-2015 (135.602 alumnos/as), se partió de un muestreo aleatorio simple adecuado a los criterios de proporcionalidad y representatividad, tomando como referencia los centros escolares. La conformación de la muestra se realizó según las siguientes variables de segmentación: provincia $(A$ 
Coruña, Lugo, Ourense y Pontevedra); contexto territorial (Zona Densamente Poblada, Zona Intermedia, Zona Poco Poblada; IGE, 2015); titularidad del centro (público/privado) y curso de escolarización (de 10 a 60 de Educación Primaria).

La selección de los centros y la asignación de grupos en los cursos de referencia fueron realizadas aleatoriamente, siendo el instrumento de recogida de información utilizado un cuestionario elaborado ad hoc para las familias (padres/tutores-madres/tutoras), entregado personalmente en el aula al alumnado de Educación Primaria por miembros del equipo investigador durante el transcurso de la jornada lectiva y solicitándoles a los niños y niñas la colaboración de sus progenitores/tutores en el estudio.

En total se entregaron 3400 cuestionarios, quedando la muestra configurada por 2037 encuestas válidas. Esta participación garantiza la representatividad para el perfil de las familias estudiado, al ser extrapolables para el conjunto de familias gallegas con hijas e hijos escolarizados en Primaria, quedando fijado el nivel de error en el 2,2\% y el nivel de confianza en el $95,5 \%$. El 78,1\% de los cuestionarios fueron cumplimentados por las madres/tutoras y el $19,8 \%$ por los padres/tutores, siendo los restantes respondidos por madre y padre $(0,3 \%)$, por otras personas $(0,3 \%)$ y en un $1,4 \%$ de los casos no se indicó quién había dado respuesta.

Los cinco bloques temáticos de los que consta el cuestionario se refieren a: datos personales; conciliación; tiempos compartidos y tiempos libres; recursos y servicios comunitarios; ocio, actividad física y salud. Los ítems empleados para la elaboración de este trabajo son:

- Hijos/as con discapacidad, trastorno y/o necesidad educativa: pertenece al bloque temático de "datos personales", haciendo referencia a si alguno de los hijos e hijas de la persona que rellena el cuestionario tiene algún tipo de necesidad específica de apoyo educativo (pregunta 10).

- Factores que dificultan conciliar la vida laboral, familiar y laboral: forma parte del bloque denominado "conciliación", indicando -en una escala tipo Likert (donde 1 se corresponde con "nada" y 4 con "mucho")- los factores que dificultan a las familias compaginar su vida laboral, familiar y personal (pregunta 13).

- Alternativas que facilitarían la organización de la vida diaria: se incluye en el bloque temático "recursos y servicios comunitarios", mostrando las alternativas que facilitarían a las familias la organización de su vida diaria en función de sus necesidades familiares (pregunta 19), recogiéndose las respuestas mediante una escala tipo Likert en la que el valor 1 se refiere a que no se perciben como necesarias y el 4 a que se necesitan mucho.

El tratamiento y análisis de los datos se ha llevado a cabo con el programa informático IBM SPSS Statistics (versión 20.0 para Windows). Así, para determinar las características de la muestra se ha realizado un análisis descriptivo, basado en porcentajes y medidas de tendencia central (medias y desviaciones típicas); y para la comparación entre variables se ha optado por la prueba Chi Cuadrado de Pearson.

\section{Resultados}

En consonancia con los objetivos que orientan este trabajo, los resultados obtenidos se agrupan en dos bloques temáticos diferenciados: por un lado, las barreras y obstáculos ("sombras") que las familias perciben en la conciliación de sus tiempos cotidianos; $y$, por otro, los aspectos favorecedores y alternativas ("luces") que consideran necesarias para la atención de sus hijas e hijos en tanto que contribuyen a la organización de su vida diaria.

\subsection{Dificultades percibidas en torno a la conciliación de los tiempos cotidianos de las familias}

A continuación, se exponen los principales resultados obtenidos en relación con los diversos factores que dificultan compaginar la vida personal, familiar y laboral de las familias gallegas con hijos/ as escolarizados en Educación Primaria ( $n=2037$ ), tratando de conocer si existen diferencias respecto a la influencia de estos factores en la conciliación de las familias del alumnado con NEAE $(n=127)$ y aquellas cuyos hijos/as no presentan dichas necesidades ( $n=1882$ ).

Partiendo de una interpretación de los resultados en la que la medida "nada" se agrupa bajo la denominación "no dificulta" la conciliación y las restantes ("poco", "bastante" y "mucho") "sí dificulta", se observa (véase gráfico 1) que un alto porcentaje de las familias participantes en la investigación ( $n=2037$ ) señala que los factores que dificultan en mayor medida poder compaginar la vida personal, familiar y laboral son los "trabajos domésticos" (76,1\%), el "horario de trabajo" (74,1\%), las "responsabilidades laborales" (67,9\%) y los "horarios extraescolares" (67,5\%). Por el contrario, aquellos factores que las madres y padres indican -con valores más elevados- que no les dificultan la armonización de sus tiempos cotidianos son la "atención a personas dependientes" (60,7\%), los "cuidados derivados del nivel de salud" (47,1\%), "otros desplazamientos" (45,7\%), los "horarios 
comerciales" $(43,4 \%)$ y los "desplazamientos al lugar de trabajo" (43,2\%). Cabe destacar que en todos los factores se obtuvo más de un $5 \%$ de NS/
NC, llegando en alguno de ellos a los siete puntos porcentuales.

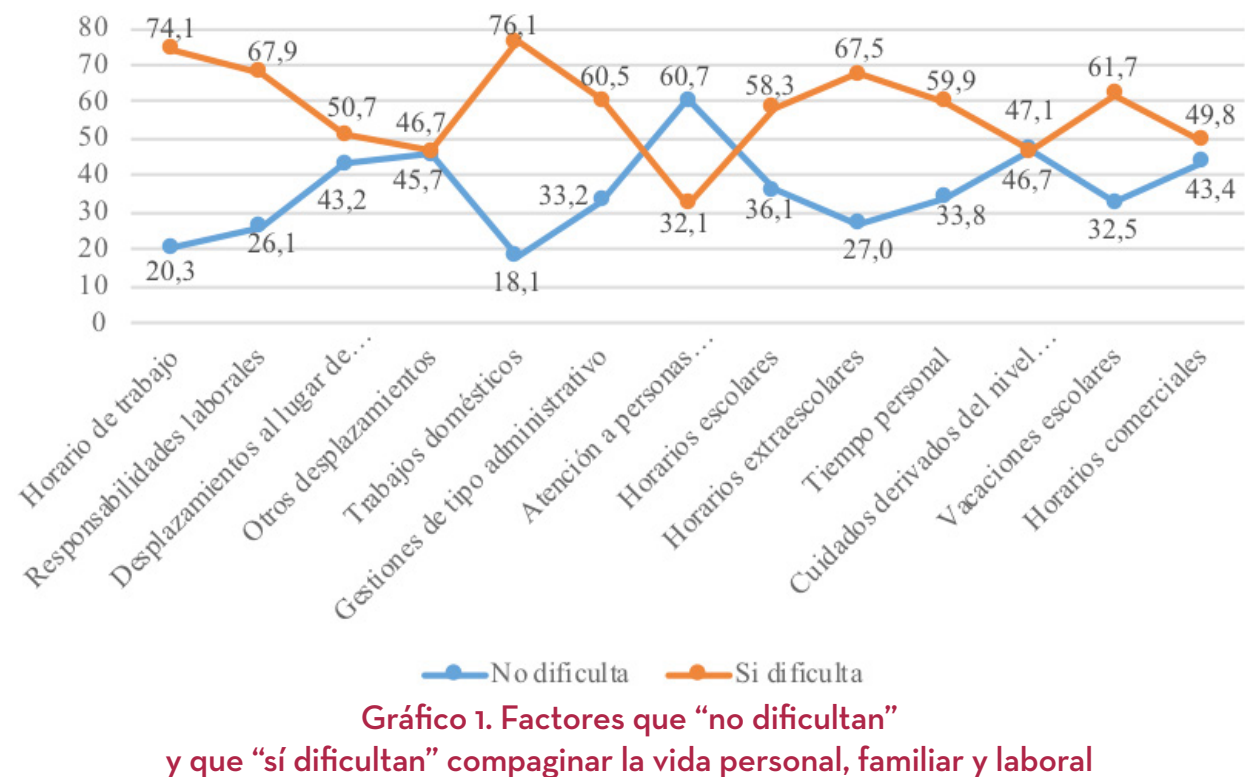

En cuanto a la opinión de las familias, en función de si su hijo/a presenta o no necesidades específicas de apoyo educativo, se constata que existe una relación de dependencia significativa entre el hecho de tener un hijo/a con o sin NEAE y los factores recogidos en el cuestionario como aspectos que podrían dificultar la conciliación de los tiempos cotidianos; exceptuando el ítem "horario de trabajo".

En general, se observa la misma tendencia entre los dos grupos de familias -con o sin hijos/as con NEAE- (véase tabla 1), siendo los factores que no dificultan "nada" la armonización de los tiempos cotidianos los vinculados con la salud propia o de la familia -mediante cuidados y atención-, así como los desplazamientos (ya sea al trabajo $\mathrm{u}$ a otros lugares) y los horarios comerciales. Sin embargo, existen ciertas diferencias porcentuales entre ambos grupos, pues las familias con menores sin NEAE señalan en un porcentaje mayor -excepto en los factores "responsabilidades laborales" $\left(x_{8}^{2}=21,006 ; p<0,05\right)$ y "horarios comerciales" $\left(x^{2}=23,914 ; p<0,05\right)$ - que los aspectos mencionados no les dificultan "nada" la conciliación. Las diferencias porcentuales van desde los 3,5 puntos respecto al factor "desplazamientos al lugar de trabajo" ( $\left.x^{2}=23,835 ; p<0,05\right)$ y los 10,4 y 8,3 relativos a "atención a personas dependientes" $\left(x_{8}^{2}=\right.$ 39,003; $p<0,05)$ y "cuidados derivados del nivel de salud" ( $\left.x_{8}^{2}=24,926 ; p<0,05\right)$, respectivamente. 


\begin{tabular}{|l|c|c|c|c|c|c|}
\hline \multicolumn{2}{|c|}{ Tabla 1. Factores que dificultan la conciliación de los tiempos cotidianos de las familias } \\
\hline & \multicolumn{3}{|c|}{$\begin{array}{c}\text { Familias con hijos/as } \\
\text { con NEAE }\end{array}$} & \multicolumn{3}{c|}{$\begin{array}{c}\text { Familias con hijos/as } \\
\text { sin NEAE }\end{array}$} \\
\cline { 2 - 8 } & Nada & Poco & $\begin{array}{c}\text { Bastante } \\
\text { Mucho }\end{array}$ & Nada & Poco & $\begin{array}{c}\text { Bastante } \\
\text { Mucho }\end{array}$ \\
\hline Horario de trabajo & 26,8 & 23,6 & 41,0 & 19,8 & 28,7 & 46,3 \\
\hline Responsabilidades laborales & 31,5 & 28,3 & 29,9 & 25,7 & 29,6 & 39,2 \\
\hline Desplazamientos al lugar de trabajo & 40,2 & 32,3 & 18,1 & 43,7 & 30,7 & 20,1 \\
\hline Otros desplazamientos & 39,4 & 29,9 & 18,9 & 46,2 & 28,3 & 18,4 \\
\hline Trabajos domésticos & 17,3 & 29,1 & 44,9 & 18,0 & 35,3 & 41,4 \\
\hline Gestiones de tipo administrativo & 27,6 & 42,5 & 19,7 & 33,5 & 41,7 & 19,1 \\
\hline Atención a personas dependientes & 51,2 & 13,4 & 23,6 & 61,6 & 18,4 & 13,4 \\
\hline Horarios escolares & 32,3 & 34,6 & 23,6 & 36,5 & 35,6 & 22,9 \\
\hline Horarios extraescolares & 21,3 & 29,1 & 39,4 & 27,4 & 34,9 & 32,8 \\
\hline Tiempo personal & 29,1 & 29,9 & 29,9 & 34,2 & 34,7 & 25,4 \\
\hline Cuidados derivados del nivel de salud & 39,4 & 29,1 & 22,0 & 47,7 & 30,8 & 15,8 \\
\hline Vacaciones escolares & 32,3 & 22,8 & 33,1 & 32,6 & 27,6 & 34,8 \\
\hline Horarios comerciales & 22,8 & 20,5 & 43,3 & 32,6 & 18,0 \\
\hline
\end{tabular}

El hecho de que los valores relativos a la medida "nada" sean mayores en el caso de las familias del alumnado sin NEAE en todos los ítems -a excepción de los relativos a "responsabilidades laborales" y "horarios comerciales"- revela que a estas madres y padres los factores señalados les dificultan en menor grado la conciliación de su vida personal, familiar y laboral.

Respecto a los aspectos que tienen poca incidencia en la conciliación de los tiempos, cabe destacar que aproximadamente un $42 \%$ de cada grupo de familias (con y sin NEAE) perciben que las "gestiones de tipo administrativo" $\left(x_{8}^{2}=24,718\right.$; $p<0,05)$ les dificultan "poco" la conciliación; siendo el único caso en el que esta medida supera los valores porcentuales de las opciones "nada" y "bastante-mucho".

En cuanto a aquellos factores que dificultan la conciliación de manera notoria ("bastante-mucho"), ambos grupos de familias coinciden en señalar los "trabajos domésticos" $\left(x_{8}^{2}=19,115 ; p<0,05\right)$ y las "vacaciones escolares" $\left(x_{8}^{2}=39,529 ; p<0,05\right)$. De las familias de hijos/as con NEAE casi un $45 \%$ considera que las tareas del hogar les dificultan "bastante-mucho" compaginar su vida personal, familiar y laboral, seguido de un 39,4\% que indica los "horarios extraescolares" $\left(x_{8}^{2}=22,018 ; p<0,05\right)$ y un $33,1 \%$ los períodos vacacionales de sus hijos/as. En la misma línea, un $41,4 \%$ de las familias del alumnado sin NEAE percibe que son las responsabilidades domésticas las que obstaculizan "bastante-mucho" la armonización de sus tiempos cotidianos, seguido del 39,2\% que indica las "responsabilidades laborales" $\left(x_{8}^{2}=21,006 ; p<0,05\right)$ y el $34,8 \%$ que señala las vacaciones marcadas por el calendario escolar.

Otra tendencia que se observa es que las familias del alumnado con NEAE indican en un porcentaje mayor que no saben o no contestan (NS/ $\mathrm{NC}$ ) en qué medida repercuten diversos factores en la conciliación de sus tiempos cotidianos. En este sentido, el valor más elevado en la opción NS/NC señalado por los progenitores del alumnado con NEAE es del $11,8 \%$ relativo a: "otros desplazamientos"; "atención a personas dependientes"; "vacaciones escolares" y "horarios comerciales", frente al 7,1\% relativo a "otros desplazamientos" de las familias del alumnado sin NEAE.

El hecho de que las madres y padres de hijos/as con NEAE indiquen, en la mayor parte de los factores, valores porcentuales más elevados en la medida "nada" que en la relativa a "bastante-mucho" puede interpretarse como un dato en cierto modo positivo. No obstante, según Martínez-Figueira, Varela y De Valenzuela (2016), la 
percepción de estas familias en relación a la armonización de su vida personal, familiar y laboral es negativa; dado que un $72,4 \%$ manifiesta que el tiempo del que dispone para sí mismo no es suficiente y casi un $64 \%$ señala que tiene dificultades para conciliar su vida personal, familiar y laboral. De ahí que las diferencias porcentuales señaladas

\subsection{Alternativas percibidas en torno a la conciliación de los tiempos cotidianos de las familias}

Seguidamente se presentan los datos relativos a la percepción de las familias participantes ( $\mathrm{n}=2037$ ) sobre la medida en que diversas alternativas facilitarían la organización de su vida personal, familiar y laboral, tratando de vislumbrar si existen diferencias respecto a la influencia de estos factores en la conciliación de las familias del alumnado con NEAE $(n=127)$ y $\sin$ NEAE ( $n=1882)$. Las respuestas se muestran agrupadas en dos categorías: "no se necesitan" (contempla la opción "no las necesito") y "sí se necesitan", que incluye las medidas "las necesito poco", "las necesito bastante" y "las necesito mucho". puedan deberse a la influencia de otros aspectos en la organización de los tiempos personales, familiares y laborales.

Como se observa en el gráfico 2, las tres alternativas que las familias consideran más necesarias, expresadas en los valores porcentuales más altos, son las "actividades extraescolares" (73,5\%), el "horario escolar sólo de mañana" $(70,4 \%)$ y los "servicios municipales" (69,5\%). Por su contra, aquellas ayudas que no se consideran necesarias son el "programa de madrugadores" (71,7\%), el "transporte escolar" (67,4\%), la "apertura de la escuela en fines de semana" (63,2\%), así como el "horario escolar de mañana y tarde" (62,7\%). Por último, ha de mencionarse que el servicio de "comedor escolar" cuenta con una opinión dividida, situándose en un $52,3 \%$ las familias que lo consideran necesario y en un $44,7 \%$ aquellas que afirman no necesitarlo.

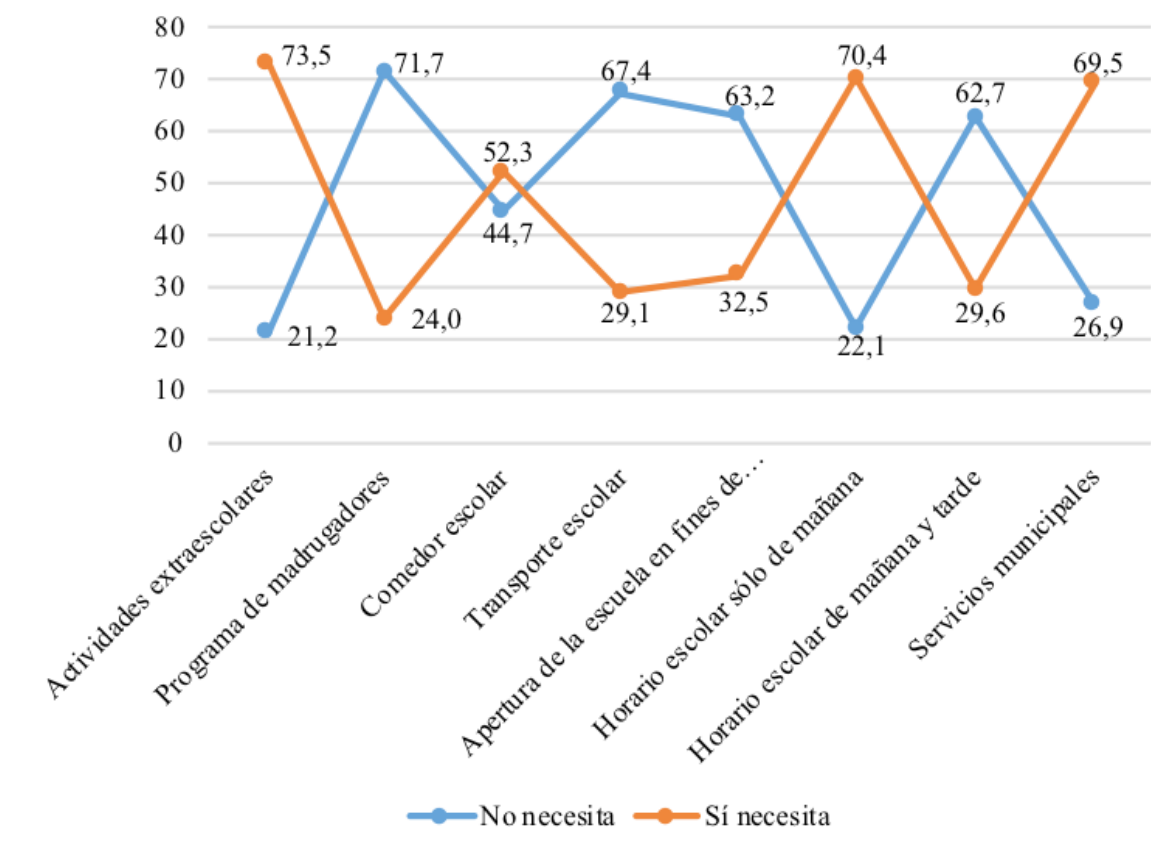

Gráfico 2. Recursos y servicios que "no necesitan" y que "sí necesitan" las familias para compaginar la vida personal, familiar y laboral.

Respecto a la opinión de las familias en función de si su hijo/a presenta, o no, necesidades específicas de apoyo educativo, los resultados evidencian que existe una relación de dependencia significativa en cuatro de las ocho alternativas señaladas en el cuestionario como servicios o recursos que pueden favorecer la organización de sus tiempos. Dicha información queda recogida en la tabla 2, agrupada en tres categorías de respuesta: "nada" (que se corresponde con la opción "no las necesito"), "poco" (que se refiere a la medida "las necesito poco") y "bastante-mucho" (que incluye las opciones de respuesta "las necesito bastante" y "las necesito mucho"). 
Tabla 2. Alternativas que facilitarían la conciliación de los tiempos cotidianos de las familias

\begin{tabular}{|l|c|c|c|c|c|c|}
\hline & \multicolumn{3}{|c|}{ Familias con hijos/as } & \multicolumn{3}{c|}{ Familias con hijos/as } \\
& \multicolumn{2}{|c|}{ son NEAE NEAE } & \multicolumn{2}{c|}{$\begin{array}{c}\text { Bastante } \\
\text { Mucho }\end{array}$} \\
\cline { 2 - 8 } & Nada & Poco & $\begin{array}{c}\text { Bastante } \\
\text { Mucho }\end{array}$ & Nada & Poco & 34,0 \\
\hline Actividades extraescolares & 19,7 & 40,2 & 34,7 & 21,3 & 39,6 & 34,0 \\
\hline Programa de madrugadores & 66,9 & 12,6 & 10,2 & 72,0 & 11,5 & 12,7 \\
\hline Comedor escolar & 34,6 & 16,5 & 44,1 & 45,4 & 15,4 & 36,4 \\
\hline Transporte escolar & 61,4 & 6,3 & 25,9 & 68,0 & 6,4 & 22,2 \\
\hline Apertura escuela fin de semana y vacaciones & 60,6 & 11,8 & 21,2 & 63,5 & 13,1 & 19,5 \\
\hline Horario escolar sólo de mañana & 18,9 & 14,2 & 58,2 & 22,3 & 10,2 & 60,2 \\
\hline Horario escolar de mañana y tarde & 50,4 & 10,2 & 26,0 & 63,5 & 10,6 & 18,7 \\
\hline Servicios municipales & 22,8 & 34,6 & 37,0 & 27,1 & 30,8 & 38,7 \\
\hline
\end{tabular}

En líneas generales, se evidencia una tendencia similar entre los dos grupos de familias -con hijas/os con NEAE ( $n=127)$ y sin NEAE ( $n=1882)$-, pues ambos manifiestan prácticamente la misma valoración sobre la necesidad percibida respecto a las alternativas señaladas.

En cuanto a la medida que alude a que no se necesitan "nada" los servicios y recursos, todos los valores porcentuales son más elevados en el caso de las familias del alumnado sin NEAE; entendiéndose así que este grupo de familias los perciben como menos útiles para la conciliación que aquellas que tienen hijas/os con NEAE. El "programa de madrugadores" $\left(x_{8}^{2}=17,695 ; p<0,05\right)$ es el servicio que ambos grupos coinciden en indicar con valores más elevados que no lo necesitan "nada"; un 66,9\% de las familias del alumnado con NEAE y un $72 \%$ de aquellas cuyos hijos/as no presentan dichas necesidades. Las mayores diferencias porcentuales entre ambos grupos de familias se registran en las alternativas "horario escolar de mañana y tarde" $\left(x_{8}^{2}=21,070 ; p<0,05\right)$ y "comedor escolar" ( $\left.x_{8}^{2}=17,564 ; p<0,05\right)$, con 13,1 y 10,8 puntos porcentuales de diferencia respectivamente.

Respecto a los servicios y recursos que se valoran como "poco" necesarios, las "actividades extraescolares" y los "servicios municipales" $\left(x_{8}^{2}=\right.$ 15,810; $p<0,05)$, son las dos alternativas que registran mayores valores porcentuales. Cabe destacar que, de las alternativas analizadas, la opción "actividades extraescolares" es la única que registra porcentajes más elevados en la medida "poco" que en las dos restantes ("nada" y "bastante-mucho").
En cuanto a las alternativas que facilitarían "bastante-mucho" la conciliación, las familias del alumnado con NEAE registran valores porcentuales más elevados en cinco de los ocho ítems. El servicio de "comedor escolar" $\left(x_{8}^{2}=17,564 ; p<0,05\right)$ y que los colegios tengan un "horario escolar de mañana y tarde" $\left(x_{8}^{2}=21,070 ; p<0,05\right)$ son las dos alternativas respecto a las que existen mayores diferencias porcentuales entre ambos grupos de familias; en concreto, de 7,7 y 7,3 puntos respectivamente. Así, las familias con hijos/as con NEAE indican en un porcentaje mayor que disponer de estas dos medidas facilitaría "bastante-mucho" la organización de su vida diaria. En referencia a que las escuelas dispongan de un "horario escolar de mañana y tarde", Ilama la atención el elevado porcentaje que registra la opción NS/NC en los dos grupos: un $13,2 \%$ de las familias con hijos/as con NEAE y un 7,2\% de los progenitores del alumnado $\sin$ NEAE.

Con todo, que los centros educativos tengan un "horario escolar sólo de mañana" es la alternativa que en un porcentaje más elevado de las familias del alumnado con y sin NEAE consideran que facilitaría "bastante-mucho" la conciliación de sus tiempos cotidianos $(58,2 \%$ y $60,2 \%$, respectivamente). Le siguen el $44,1 \%$ y el $37 \%$ relativo a la percepción de las familias del alumnado con NEAE sobre el "comedor escolar" y los "servicios municipales", respectivamente y en el caso de las familias cuyos hijos/as no presentan NEAE, el $36,4 \%$ y el $38,7 \%$ que también alude a estas dos alternativas de forma respectiva. 


\section{Discusión y conclusiones}

A partir de los datos presentados, se constata que los obstáculos que han de afrontar las familias con hijos e hijas con NEAE para la organización de los tiempos son mayores que los de las familias con hijos e hijas sin NEAE, destacando la incidencia de las tareas domésticas (limpieza, alimentación...), de los horarios extraescolares (en el colegio y en otros espacios) y de las vacaciones escolares en la conciliación. Las responsabilidades laborales también influyen, siendo menor su impacto en las familias del alumnado sin NEAE, aun cuando son un condicionante importante en la organización de su vida cotidiana.

En cuanto a los impedimentos mencionados, cabe destacar las desigualdades existentes en la realización de las tareas domésticas debido -en gran medida- a la falta de una distribución equitativa de este tipo de quehaceres entre hombres y mujeres; a lo que se une la dedicación temporal que exige la crianza y educación de un hijo/a con NEAE. Si bien la atención educativa de la infancia continúa siendo una tarea feminizada (téngase en cuenta que el $78,1 \%$ de los cuestionarios en la presente investigación fueron cumplimentados por las madres y solo el 19,8\% por los padres), también la asunción de las responsabilidades domésticas se mantiene como un asunto propio del género femenino.

Desde esta perspectiva, el estudio de Calvo y Picontó (2014) revela que la conciliación es "cosa de mujeres" (son quienes se encargan de esta tarea) y está centrada fundamentalmente en "el cuidado de los hijos/as". Algunas evidencias de ello son que de las personas que participaron en su investigación, casi la totalidad (95\%) de las excedencias solicitadas para el cuidado de familiares fueron requeridas por mujeres y que las peticiones sobre derechos de conciliación aluden principalmente a la maternidad (embarazo, lactancia, cuidado de los hijos/as, etc.). De ahí que el ejercicio de los derechos de conciliación refuerce el papel de la mujer como cuidadora, pues facilitar la compatibilización de las distintas esferas vitales no ha derivado en una revisión del contrato entre los géneros establecido en el patriarcado (Alcañiz, 2015).

En el estudio que se ha desarrollado destacan los desajustes temporales que se producen entre la jornada laboral de los progenitores y el calendario y horario escolar, siendo en las familias del alumnado con NEAE los trabajos domésticos, los tiempos de trabajo y extraescolares y los periodos vacacionales los más conflictivos para la conciliación. De las opciones orientadas a favorecer la armonización de los tiempos cotidianos, los recursos y servicios que se identifican como más necesarios son un horario escolar sólo de mañana, que las escuelas cuenten con comedor escolar, así como los servicios municipales (bibliotecas, ludotecas, escuelas deportivas, etc.) y actividades extraescolares.

Según Varela, Varela y Lorenzo (2016), el tiempo escolar resulta tan relevante para las rutinas familiares que, habitualmente, la organización de la vida cotidiana se realiza en función de los diferentes servicios y programas que ofertan las escuelas. Es un hecho que

los cronómetros de la escuela demandan a cada familia acompasarse a sus ritmos y exigencias, al alumnado supeditarse a una organización temporal escolar obsoleta y anacrónica, más atenta a los requerimientos administrativos y exigencias laborales de los adultos que a las necesidades, potencialidades y derechos de las infancias (Morán \& Cruz, 2011, p. 89).

De este modo, considerando las dificultades manifestadas por las familias participantes en esta investigación respecto a la conciliación de los tiempos cotidianos, resulta paradójico que se identifique como necesario el horario escolar sólo de mañana -extendido en el contexto español y en el gallego-, siendo percibido más positivamente que el de mañana y tarde; cuando el horario laboral más habitual de las familias es el de jornada partida (40,2\%), seguido de la jornada continua en horario fijo de mañana (28,6\%), tal como sugiere el estudio realizado por Nogareda, Nogareda y Solórzano (2014). Son múltiples los autores (Fernández Enguita, 2001; Caride, 2005) que desde hace ya varias décadas han venido afirmando que la implantación de la jornada única en la escuela pública deriva en mayor medida de los intereses corporativos del profesorado y de algunas familias, y no de la innovación educativa y de la adaptación de los tiempos escolares a los ritmos cronopsicobiológicos de la infancia. En este sentido, Sintes (2015) señala la urgente necesidad de superar la dialéctica entre jornada partida o continua para pasar a un modelo centrado en el alumnado y en sus necesidades educativas.

Este desajuste de tiempos, reflejado en una escuela que no se adapta a los ritmos de la infancia y unos horarios de trabajo que dificultan el ejercicio de la paternidad/maternidad, obliga a las familias a reclamar servicios que custodien a los niños y niñas fuera del horario lectivo mientras cumplen con sus responsabilidades laborales. Un tiempo extraescolar que puede convertirse en un componente importante de las desigualdades educativas, al ser las familias con un nivel de estudios alto, residentes en el contexto urbano, de barrios acomodados, etc., quienes suelen disponer 
de más posibilidades de acceso a este tipo de iniciativas, mientras que el alumnado que no tiene la oportunidad de disfrutar de ciertos servicios y recursos durante las tardes se ve perjudicado, al no poder participar en experiencias de ocio enriquecedoras, desde una perspectiva tanto individual (autorrealización, formación, bienestar, recreación, etc.) como comunitaria (participación, cohesión social, etc.).

Respecto a los apoyos que reciben las familias, investigaciones precedentes (Heiman, 2002; Felizardo, Ribeiro, Cardoso \& Campos, 2014) indican que a pesar de la amplitud de necesidades que presentan los progenitores con hijos/as con NEAE, su percepción acerca de las ayudas con las que cuentan parece ser menos satisfactoria. Desde esta perspectiva, se apunta que los servicios y programas deben contemplar formas de acompañar a las familias que incluyan aspectos referidos a la especificidad de las dificultades de los menores y temas relacionados con el aprendizaje, la prevención y la inclusión social. Considerando las limitaciones del presente estudio, resulta de interés el desarrollo de nuevas investigaciones en este ámbito con la finalidad de conocer si en función del tipo de NEAE existen diferencias en las necesidades y demandas de las familias respecto a la conciliación de los tiempos cotidianos, incluyendo una perspectiva cualitativa que permita profundizar en las percepciones y en los discursos tanto de los progenitores como de la infancia.

Por último, con los hallazgos obtenidos, cabe afirmar que aún estamos lejos de responder a la idea de inclusión social formulada por la UNESCO (2007) referida al modo de favorecer la equidad y contribuir a una mayor cohesión social, procurando dar respuesta a las necesidades de toda la ciudadanía mediante una mayor participación en la escuela, en las actividades culturales y comunitarias, minimizando así las trayectorias de exclusión social (Parrilla, 2013). Como señala Dyson (2001), la inclusión simplemente denota una serie de amplios principios de justicia social, siendo necesario desarrollar propuestas en diversos contextos, tanto escolares como sociales, que tengan en consideración las necesidades educativas. En este marco, el desafío de la Pedagogía Social respecto a la conciliación de los tiempos cotidianos de las familias con hijos/as con NEAE se fundamenta en lograr la democratización del uso de los tiempos y la satisfacción de las personas con su vida, así como en transformar determinadas estructuras que impiden valorar las tareas de cuidado en un plano de igualdad haciendo corresponsable al conjunto de la sociedad.

\section{Referencias bibliográficas}

Alcañiz, M. (2015). Género con clase: la conciliación desigual de la vida laboral y familiar. RES. Revista Española de Sociología, 23, 29-55.

Algood, C. L., Harris, C., \& Sung, J. (2013). Parenting Success and Challenges for Families of Children with Disabilities: An Ecological Systems Analysis. Journal of Human Behavior in the Social Environment, 23(2), 126-136. DOI: $10.1080 / 10911359.2012 .747408$

Browne, G., \& Bramster, P. (1998). Stress and the quality of life of the parents of young people with intelectual disability. Journal of Psychiatric and Mental Health Nursing, 5, 415-421.

Caballo, M. B., Caride, J. A., \& Meira, P. A. (2011). El tiempo como contexto y pretexto educativo en la sociedad red. Educación social: revista de intervención socioeducativa, 47, 11-24.

Caballo, M. B., Gradaílle, R., \& Merelas, T. (2012). Servicios socioeducativos y corresponsabilidad en la conciliación de los tiempos familiares: situación de la infancia en la Galicia urbana. Pedagogía Social. Revista Interuniversitaria, 20, 179-2O2.

Calvo, M., \& Picontó, T. (eds.) (2014). Valoración de las medidas de conciliación de la vida familiar y personal y la vida laboral según la regulación de la Ley Orgánica 3/2007 para la igualdad efectiva de mujeres y hombres. Madrid: Consejo General del Poder Judicial.

Caride, J. A. (dir.) (2005). A xornada escolar de sesión única en Galicia: avaliación da súa implantación e desenvolvemento nos centros de Educación Infantil, Primaria e Públicos Integrados. Santiago de Compostela: Xunta de Galicia.

Córdoba-Andrade, L., Gómez-Benito, J., \& Verdugo-Alonso, M. A. (2008). Calidad de vida familiar en personas con discapacidad: un análisis comparativo. Universitas Psichologica, 7(2), 369-383.

Díaz, L., Gil, A., \& Moral, D. (2010). La experiencia de familias que viven la discapacidad intelectual. Revista Educación y Futuro, 23, 81-89

Dyson, A. (2001). Dilemas, contradicciones y variedades de la inclusión. En M. Verdugo y F. Jordán de Urríes (Eds.), Apoyos, autodeterminación y calidad de vida (pp. 145-160). Salamanca: Amarú.

Felizardo, S. A., Ribeiro, E. S., Cardoso, A. P., \& Campos, S. (2014). Família e bem-estar: contributos para uma educação inclusiva. International Journal of Developmental and Educational Psychology. INFAD, Revista de Psicología, 7(6), 375-380. DOI: 10.17060/ijodaep.2014.n1.v6.75 
Fernández Enguita, M. (2001). La jornada escolar. Análisis y valoración de los procesos, los efectos y las opciones de la implantación de la jornada continua. Barcelona: Ariel.

Gerstein, E. D., Crnic, K. A., Blacher, J. J., \& Baker, B. L. (2009). Resilience and the course of daily parenting stress in families of young children with intellectual disabilities. Journal of Intellectual Disability Research, 53, 981-997.

Gómez, V., \& Jiménez, A. (2015). Corresponsabilidad familiar y el equilibrio trabajo-familia: medios para mejorar la equidad de género. Polis, 40, 1-18.

Heiman, T. (2002). Parents of children with disabilities: Resilience, coping and future expectations. Journal of Developmental and Physical Disabilities, 14(2), 159-171. DOI: 10.1023/A:1015219514621

IGE (2015). Panorama rural-urbano. Instituto Galego de Estatística. Recuperado de http://www.ige.eu/web/mostrar_ seccion.jsp?idioma $=$ gl\&codigo $=0701$

Jung, S., \& Appelbaum, E. (2010). Work-Life Balance in extraordinary circumstances. Journal of Women, Politics \& Policy, 31(4), 313-333. DOI:10.1080/1554477X.2010.517156.

Küçük, E. E., \& Küçük, D. (2017). Life satisfaction and psychological status of mothers with disabled children: a descriptive study. Community mental health journal, mar 31. DOI: 10.1007/s10597-017-0135-6.

Lee, Y. J., Park, H., \& Recchia, S. (2015). Embracing each other and growing together: redefining the meaning of caregiving a child with disabilities. Journal of Child \& Family Studies, 24(12), 3662-3675.

Liñán, G. (2013). El impacto de una persona con discapacidad intelectual en la conciliación familiar en el ámbito personal, familiar y laboral. Universidad de Zaragoza: Zaragoza.

Maganto, J. Ma., \& Etxeberria, J. (2012). La corresponsabilidad como base de la conciliación. En J. Ma. Maganto, Programa Concilia. Concilia en tu vida. La conciliación de la vida personal, familiar y académico-laboral en adolescentes, jóvenes y adultos (pp. 163-216). Madrid: Pirámide.

Martínez-Figueira, M. E., Varela, L., \& De Valenzuela, A. L. (2016). Diversidad y conciliación en Galicia. Un desafío para la educación inclusiva. Prisma Social. Revista de investigación social, 16, 111-155.

Merino, I. (2015). La conciliación laboral, personal y familia; análisis desde una doble vertiente: comunitaria y nacional. Revista jurídica de Castilla y León, 36, 1-45.

Mingo, M. L., \& Escudero, A. (2008). Calidad de vida de los menores con discapacidad. Análisis de la situación en España de la inclusión de los menores: atención temprana; atención educativa; protección de la familia; seguridad social y servicios sociales; conciliación de la vida laboral y familiar. En L.C. Pérez Bueno (dir.), Los menores con discapacidad en España (pp. 201-330). Madrid: Ediciones Cinca.

Morán, M. C., \& Cruz, L. (2011). Vida cotidiana, tempos escolares y derechos de la infancia. Educación Social. Revista de intervención socioeducativa, 47, 84-94.

Moreno, S. (2009). Las políticas de conciliación de la vida laboral, familiar y personal: ¿éxito o fracaso? Aequalitas. Revista jurídica de igualdad de oportunidades entre mujeres y hombres, 25, 46-50.

Nogareda, C., Nogareda, S., \& Solórzano, M. (2014). Jornadas y horarios de trabajo. Barcelona: Centro Nacional de Condiciones de Trabajo.

Parrilla, A. (2013). Presentación. Equidad e Innovación en la investigación educativa: reflexiones y aportaciones desde la red de investigación CIES. Revista de Investigación en Educación, 11(3), 7-13.

Park, J., Turnbull, A., \& Turnbull, R. (2002). Impacts of poverty on quality of life in families of children with disabilities. Exceptional Children, 68, 151-170.

Rivero, A. (dir.) (2005). Conciliación de la vida familiar y la vida laboral: Situación actual, necesidades y demandas (Informe de resultados). Madrid: Instituto de la Mujer (Ministerio de Igualdad).

Rubio, D., Berg-Weger, M., \& Tebb, S. (1999). Assessing the validity and reliability of well-being and stress in family caregivers. Social Work Research, 23(1), 54-66.

Sánchez, E. (2011). Conciliar per educar. Informes breus, 34. Barcelona: Fundació Jaume Bofill.

Schalock, R., \& Verdugo, M. (2002). The concept of quality of life in human services: A handbook for human service practitioners. Washington, DC: American Association on Mental Retardation.

Sintes, E. (2015). Escola a temps complet. Cap a un model d'educació compartida. Barcelona: Fundació Jaume Bofill.

Trute, B., Hiebert-Murphy, D., \& Levine, K. (2007). Parental appraisal of the family impact of childhood developmental disability: times of sadness and times of joy. Journal of Intellectual and Developmental Disability, 32(1), 1-9.

UNESCO (2007). El derecho a una educación de calidad para todos en América latina y el Caribe. REICE - Revista Electrónica Iberoamericana sobre Calidad, Eficacia y Cambio en Educación, 5(3), 1-21.

Varela, L., Varela, L., \& Lorenzo, J. J. (2016). La escuela y la conciliación de los tiempos cotidianos de las familias del alumnado de Educación Primaria en Galicia. Bordón. Revista de Pedagogía, 68(4), 179-194. DOI: 10.13042/ Bordon.2016.48602.

Vives, L. (2007). Conciliar la vida laboral y familiar. SIGLO CERO Revista Española sobre Discapacidad Intelectual, 38, 29-44.

Weiss, K., Marvin, R., \& Pianta, R. (1997). Ethnographic detection and description of family strategies for child care: Applications to the study of cerebral palsy. Journal of Pediatric Psychology, 22, 263-278. 


\section{CÓMO CITAR ESTE ARTÍCULO}

Varela, L., Martínez-Figueira, M.E., \& Valenzuela, A. (2018). Luces y sombras en torno a la conciliación de las familias con hijos e hijas con NEAE: un estudio en Galicia. Pedagogía Social. Revista Interuniversitaria, 32 45-57. DOI:10.7179/PSRI_2018.32.04

\section{DIRECCIÓN COMPLETA DE LOS AUTORES}

Laura Varela Crespo. Universidad de Santiago de Compostela. Departamento de Pedagogía y Didáctica. Facultad de Ciencias de la Educación-Campus vida. Rúa Prof. Vicente Fráiz Andón, s/n. 15782, Santiago de Compostela. E-mail: laura.varela@usc.es

M. Esther Martínez-Figueira. Universidad de Vigo. Departamento de Didáctica, Organización Escolar y Métodos de Investigación. Facultad de Ciencias de la Educación y del Deporte-Campus Pontevedra. A Xunqueira, s/n. 36005, Pontevedra. E-mail: esthermf@uvigo.es

Ángela L. de Valenzuela Bandín. Universidad de Santiago de Compostela. Departamento de Pedagogía y Didáctica. Facultad de Ciencias de la Educación-Campus vida. Rúa Prof. Vicente Fráiz Andón, s/n. 15782, Santiago de Compostela. E-mail: angela.devalenzuela@usc.es

\section{PERFIL ACADÉMICO}

Laura Varela Crespo. Doctora en Ciencias de la Educación, Licenciada en Pedagogía y Diplomada en Educación Social. Profesora Ayudante Doctora en el Departamento de Pedagogía y Didáctica de la Universidad de Santiago de Compostela y miembro del grupo de investigación en Pedagogía Social y Educación Ambiental (SEPA-interea). Sus principales líneas de investigación y publicación son: pedagogía-educación social, servicios sociales, tiempos educativos y sociales, pedagogía del ocio.

M. Esther Martínez-Figueira. Doctora en Ciencias de la Educación, Licenciada en Psicopedagogía y Diplomada en Educación Infantil. Profesora Titular de Universidad en el Departamento de Didáctica, Organización Escolar y Métodos de Investigación de la Universidad de Vigo y miembro del grupo CIES (Colaboración e Innovación para la Equidad Educativa y Social). Sus principales líneas de investigación y publicación giran en torno a la tecnología educativa, educación inclusiva, innovación docente y formación del profesorado.

Ángela L. De Valenzuela Bandín. Diplomada y habilitada al Grado en Educación Social por la Universidad de Santiago de Compostela (USC), con Premio Extraordinario de Diplomatura en el año 2010. Cuenta con dos Másteres Universitarios y actualmente está desarrollando una tesis doctoral que versa sobre los tiempos de ocio de los jóvenes (ex)tutelados. Es miembro del grupo de investigación en Pedagogía Social y Educación Ambiental (SEPA-interea). Sus principales líneas de investigación y publicación son infancia y juventud en situación de riesgo y/o dificultad social, pedagogía del ocio, tiempos educativos y sociales 
